DESY 11-087

ISSN 0418-9833

May 2011

\title{
Counting master integrals: integration by parts vs. differential reduction
}

\author{
Mikhail Yu. Kalmykov*, Bernd A. Kniehl \\ II. Institut für Theoretische Physik, Universität Hamburg, \\ Luruper Chaussee 149, 22761 Hamburg, Germany
}

\begin{abstract}
The techniques of integration by parts and differential reduction differ in the counting of master integrals. This is illustrated using as an example the two-loop sunset diagram with on-shell kinematics. A new algebraic relation between the master integrals of the two-loop sunset diagram that does not follow from the standard integration-by-parts technique is found.
\end{abstract}

PACS numbers: 02.30.Gp, 02.30.Lt, 11.15.Bt, 12.38.Bx

Keywords: Two-loop sunset; Generalized hypergeometric functions; Differential reduction; Multiloop calculations.

Dedicated to K.G. Chetyrkin on the occasion of his sixtieth birthday.

\footnotetext{
*On leave of absence from Joint Institute for Nuclear Research, 141980 Dubna (Moscow Region), Russia.
} 
Within dimensional regularization [1, the integration-by-parts (IBP) technique [2] (for a recent review, see Ref. [3]) is one of the most powerful tools for evaluating multiloop Feynman diagrams. However, the question how to construct algebraical, geometrical, or topological criteria for the (ir)reducibility of Feynman diagrams in general is still an open one [4]. At present, only the explicit solution of recurrence relations (see, for example, Ref. [5]), or the application of the Laporta algorithm [6], as implemented in computer programs [7], provide an answer on this question.

In a series of recent publications 891011, we addressed the problem of counting nontrivial master integrals with the help of hypergeometric representations for Feynman diagrams and the differential-reduction algorithm [12]. By detailed analysis, we thus derived the following empirical criteria for the hypergeometric representation of Feynman diagrams: If a Feynman diagram is expressible as a linear combination of Horn-type hypergeometric functions with rational coefficients, then (i) each hypergeometric function has the same number of basis elements in the framework of differential reduction [12]; and (ii) the number of nontrivial master integrals is equal to the number of basis elements of the hypergeometric functions (up to the modules of Feynman integrals expressible in terms of products of algebraic functions and $\Gamma$ functions).

These criteria were conjectured on the basis of the analysis of a variety of particular examples [8910]. A rigorous mathematical proof is still lacking. The aim of this Letter is to present a first example where the application of our criteria allow us to find an additional algebraic relation between master integrals which does not follow from the standard IBP technique. In fact, Tarasov's algorithm [5] for the reduction of two-loop propagator diagrams, as implemented in the computer packages of Ref. [13], and Laporta's algorithm [6], as implemented in the computer package of Ref. [7, fail to produce this relation.

Let us consider the two-loop self-energy sunset-type diagram $J_{012}$ with on-shell kinematics, defined as

$$
J_{012}(\sigma, \beta, \alpha)=\left.\pi^{-n} \int \frac{d^{n} k_{1} d^{n} k_{2}}{\left[\left(p-k_{1}\right)^{2}\right]^{\sigma}\left[\left(k_{1}-k_{2}\right)^{2}+M^{2}\right]^{\alpha}\left[k_{2}^{2}+m^{2}\right]^{\beta}}\right|_{p^{2}=-m^{2}},
$$

where $n=4-2 \varepsilon$ is the dimensionality of space time (see Fig. 1). Such a diagram

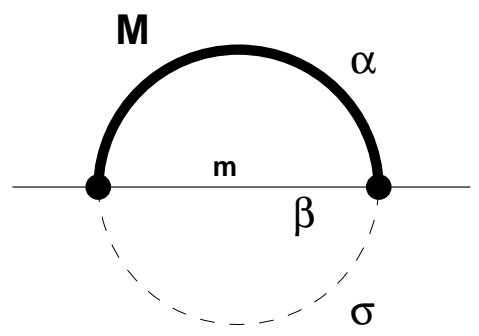

\section{J012}

Figure 1: Two-loop self-energy sunset-type diagram $J_{012}$. 
contributes to the pole mass of the top quark [14. The hypergeometric representation of this diagrams was presented in Eq. (3.12) of Ref. [14. We reproduce it here for completeness:

$$
\begin{aligned}
& J_{012}(\sigma, \beta, \alpha)=\left(M^{2}\right)^{n-\sigma-\alpha-\beta} \frac{\Gamma\left(\frac{n}{2}-\sigma\right)}{\Gamma(\sigma) \Gamma(\alpha) \Gamma(\beta) \Gamma\left(\frac{n}{2}\right)}\left[\Gamma\left(\frac{n}{2}-\beta\right) \Gamma(\alpha+\beta+\sigma-n)\right. \\
& \times \Gamma\left(\beta+\sigma-\frac{n}{2}\right){ }_{4} F_{3}\left(\begin{array}{c}
\alpha+\beta+\sigma-n, \beta+\sigma-\frac{n}{2}, \frac{\beta}{2}, \frac{1+\beta}{2} \\
1+\beta-\frac{n}{2}, \beta, \frac{n}{2}
\end{array} \mid \frac{m^{2}}{M^{2}}\right)+\left(\frac{m^{2}}{M^{2}}\right)^{n / 2-\beta} \\
& \left.\quad \times \Gamma\left(\beta-\frac{n}{2}\right) \Gamma(\sigma) \Gamma\left(\alpha+\sigma-\frac{n}{2}\right){ }_{4} F_{3}\left(\begin{array}{c}
\sigma, \alpha+\sigma-\frac{n}{2}, \frac{n-\beta}{2}, \frac{1+n-\beta}{2} \\
1+\frac{n}{2}-\beta, n-\beta, \frac{n}{2}
\end{array} \mid \frac{4 m^{2}}{M^{2}}\right)\right] .
\end{aligned}
$$

In the framework of differential reduction [9], the first hypergeometric function in Eq. (2) is expressible in terms of a Gauss hypergeometric function and a rational function $R_{1}(z)$ of $z=4 \mathrm{~m}^{2} / M^{2}$, whereas the second one is expressible in terms of a ${ }_{3} F_{2}$ function with one unit upper parameter and a rational function $R_{2}(z)$. Schematically, we have

$$
\begin{aligned}
& { }_{4} F_{3}\left(\begin{array}{c}
\alpha+\beta+\sigma-n, \beta+\sigma-\frac{n}{2}, \frac{\beta}{2}, \frac{1+\beta}{2} \\
1+\beta-\frac{n}{2}, \beta, \frac{n}{2}
\end{array} \mid z\right) \rightarrow(1, \theta) \times{ }_{2} F_{1}\left(\begin{array}{c}
I_{1}-n, \frac{1}{2}+I_{2} \\
\frac{n}{2}+I_{3}
\end{array} \mid z\right)+R_{1}(z), \\
& { }_{4} F_{3}\left(\begin{array}{c}
\sigma, \alpha+\sigma-\frac{n}{2}, \frac{n-\beta}{2}, \frac{1+n-\beta}{2} \\
1+\frac{n}{2}-\beta, n-\beta, \frac{n}{2}
\end{array} \mid z\right) \rightarrow(1, \theta) \times{ }_{3} F_{2}\left(\begin{array}{c}
1, I_{1}-\frac{n}{2}, \frac{n}{2}+\frac{1}{2}+I_{2} \\
n+I_{3}, \frac{n}{2}+I_{4}
\end{array} \mid z\right)+R_{2}(z),
\end{aligned}
$$

where $\theta=z d / d z$, the short-hand notation $(1, \theta)$ stands for $\left(P_{1}(z)+P_{2}(z) \theta\right)$, with $P_{i}$ being rational functions (see Eqs. (17) and (20) in Ref. [9]), and $I_{i}(i=1, \ldots, 4)$ are integers. According to our criteria, there are two master integrals for this diagram that are not expressible in terms of $\Gamma$ functions. However, as shown by Tarasov in Ref. [5], solving the standard IBP relations [2] yields three master integrals of this type, namely $J_{012}(1,1,1), J_{012}(1,2,1)$, and $J_{012}(1,1,2)$, which is confirmed with the help of the computer packages of Refs. [713]. Consequently, either our criteria are wrong or there exists an algebraic relation between the integrals $J_{012}(1,1,1)$, $J_{012}(1,2,1)$, and $J_{012}(1,1,2)$, possibly including some algebraic functions depending on $z$ and products of $\Gamma$ functions.

To find this relation, let us explore Eq. (2) and present the master integrals in the following form:

$$
X_{111}=A x_{1}+B y_{1}, \quad X_{121}=A x_{2}+B y_{2}, \quad X_{112}=A x_{3}+B y_{3},
$$

where

$$
\begin{aligned}
X_{\sigma \beta \alpha} & =\left(M^{2}\right)^{4-n}\left(\frac{n}{2}-1\right) J_{012}(\sigma, \beta, \alpha), \\
A & =\Gamma\left(\frac{n}{2}-1\right) \Gamma(3-n) \Gamma\left(2-\frac{n}{2}\right), \quad B=\left(\frac{z}{4}\right)^{n / 2-1} \Gamma\left(1-\frac{n}{2}\right) \Gamma\left(2-\frac{n}{2}\right), \\
x_{1} & ={ }_{2} F_{1}\left(\frac{1}{2}, a ; b ; z\right), \quad x_{2}=\frac{a-1}{z}\left[{ }_{2} F_{1}\left(\frac{1}{2}, a ; b ; z\right)-1\right], \\
x_{3} & =a_{2} F_{1}\left(\frac{1}{2}, 1+a ; b ; z\right), \quad y_{1}={ }_{3} F_{2}\left(1, \frac{n-1}{2}, c ; \frac{n}{2}, d ; z\right), \\
y_{2} & =-\frac{2}{z}(d-1)_{3} F_{2}\left(1, \frac{n-1}{2}, c ; \frac{n}{2}, d-1 ; z\right), \quad y_{3}=c_{3} F_{2}\left(1, \frac{n-1}{2}, 1+c ; \frac{n}{2}, d ; z\right),
\end{aligned}
$$


with

$$
a=3-n, \quad b=\frac{n}{2}, \quad c=2-\frac{n}{2}, \quad d=n-1,
$$

and ${ }_{p} F_{p-1}(\vec{a} ; \vec{b} ; z)$ being a hypergeometric function. Notice that the ${ }_{4} F_{3}$ functions in Eq. 2 collapse to ${ }_{3} F_{2}$ and ${ }_{2} F_{1}$ functions according to Criterion I defined in Section 2.4 of Ref. 9] because upper indices exceed lower ones by integers. Using the relations

$$
\begin{aligned}
a_{p} F_{q}(a+1, \vec{A} ; \vec{B} ; z) & =(\theta+a)_{p} F_{q}(a, \vec{A} ; \vec{B} ; z), \\
(b-1)_{p} F_{q}(\vec{A} ; b-1, \vec{B} ; z) & =(\theta+b-1)_{p} F_{q}(\vec{A} ; b, \vec{B} ; z),
\end{aligned}
$$

it is easy to obtain

$$
\begin{aligned}
(3 n-8) x_{1}+z x_{2}+2 x_{3} & =n-2, \\
(3 n-8) y_{1}+z y_{2}+2 y_{3} & =0 .
\end{aligned}
$$

This is equivalent to the following relation between master integrals:

$$
\begin{aligned}
& (3 n-8) J_{012}(1,1,1)+4 m^{2} J_{012}(1,2,1)+2 M^{2} J_{012}(1,1,2) \\
& =2\left(M^{2}\right)^{n-3} \Gamma\left(\frac{n}{2}-1\right) \Gamma(3-n) \Gamma\left(2-\frac{n}{2}\right) .
\end{aligned}
$$

Eq. (9) does not only represent a relation between Feynman diagrams, but also a relation between hypergeometric functions ${ }_{p} F_{q}$, which is newly derived from the differential reduction technique.

For $m^{2}=M^{2}$, we have

$$
(3 n-8) J_{011}(1,1,1)+6 m^{2} J_{011}(1,1,2)=2\left(m^{2}\right)^{n-3} \Gamma\left(\frac{n}{2}-1\right) \Gamma(3-n) \Gamma\left(2-\frac{n}{2}\right) .
$$

The integrals $J_{011}(1,1,1)$ and $J_{011}(1,1,2)$ with $m^{2}=M^{2}$ are master integrals of the package ON-SHELL2 [15]. Eq. (10) coincides with Eq. (4.45) of Ref. [16, where it was derived by studying the analytical coefficients of the higher-order $\varepsilon$ expansion of diagram $J_{011}(1,2,2)$ performed in Ref. [17].

According to Ref. [18], the last terms in Eqs. (9) and (10) may be identified with a two-loop bubble diagram with one massive line divided by $M^{2}(n-2)$, so that Eq. (9) is an algebraic relation between four two-loop diagrams. However, that two-loop bubble diagram, having two massless lines, cannot be obtained from the original two-loop sunset diagram with two massive lines by the contraction of lines. Consequently, Eq. (91) cannot be derived from standard IBP relations for two-loop sunset diagrams.

At this point, a comment on the structural difference between the differentialreduction and IBP approaches seems appropriate. Eq. (9) represents a linear relation between master integrals that contains an inhomogenious term involving $\Gamma$ functions on the right-hand side. However, it is clear from the structure of the IBP relations that the IBP method can only result in relations between integrals in which the prefactors are rational functions, while $\Gamma$ functions do not appear in IBP relations. This is another reason why Eq. (9) cannot be derived using the IBP method. 
In conclusion, counting the number of irreducible master integrals for the diagram defined by Eq. (11) using our criteria on the one hand and the explicit analytical solution [56] of the standard integration-by-part relations [2], as implemented in the widely used program packages 7 [13], on the other hand, we encountered a mismatch. This motivated us to established a new algebraic relation, namely Eq. (9). This is a first example where our criteria allow us to expose a new relation between master integrals of the standard integration-by-part technique.

Finally, we wish to mention that the final result of Ref. [14] does not depend on the number of master integrals. In fact, all master integrals are expressible in terms of hypergeometric functions, and the analytical coefficients of the $\varepsilon$ expansions of the latter were constructed in Ref. [19].

\section{Acknowledgments}

We are grateful to P.A. Baikov, A.I. Davydychev, A.G. Grozin, A.V. Kotikov, and O.L. Veretin for their interest in our work and for cross checking Eq. (9). We thank P.A. Baikov and A.G. Grozin for sharing with us their observation that the propagator diagrams entering Eq. (9) may be considered as special cases of more general propagator diagrams with five internal lines. This work was supported in part by the German Federal Ministry for Education and Research BMBF through Grant No. 05 HT6GUA, by the German Research Foundation DFG through the Collaborative Research Centre No. 676 Particles, Strings and the Early UniverseThe structure of Matter and Space Time, and by the Helmholtz Association HGF through the Helmholtz Alliance Ha 101 Physics at the Terascale.

\section{References}

[1] G. 't Hooft, M. Veltman, Nucl. Phys. B 44 (1972) 189.

[2] F.V. Tkachov, Phys. Lett. B 100 (1981) 65;

K.G. Chetyrkin, F.V. Tkachov, Nucl. Phys. B 192 (1981) 159.

[3] A.G. Grozin, "Integration by parts: An introduction," arXiv:1104.3993.

[4] P.A. Baikov, Phys. Lett. B 474 (2000) 385 arXiv:hep-ph/9912421;

P.A. Baikov, Phys. Lett. B 634 (2006) 325 arXiv:hep-ph/0507053;

J. Gluza, K. Kajda, D.A. Kosower, Phys. Rev. D 83 (2011) 045012 arXiv:1009.0472 [hep-th]];

O.L. Veretin, private communication.

[5] O.V. Tarasov, Nucl. Phys. B 502 (1997) 455 [arXiv:hep-ph/9703319].

[6] S. Laporta, Int. J. Mod. Phys. A 15 (2000) 5087 arXiv:hep-ph/0102033.

[7] C. Anastasiou, A. Lazopoulos, J. High Energy Phys. 07 (2004) 046 arXiv:hep-ph/0404258;

A.V. Smirnov, J. High Energy Phys. 10 (2008) 107 [arXiv:0807.3243 [hep-ph]];

C. Studerus, Comput. Phys. Commun. 181 (2010) 1293 arXiv:0912.2546 [physics.comp-ph]].

[8] M.Yu. Kalmykov, J. High Energy Phys. 04 (2006) 056 [arXiv:hep-th/0602028]. 
[9] V.V. Bytev, M.Yu. Kalmykov, B.A. Kniehl, Nucl. Phys. B 836 (2010) 129 arXiv:0904.0214 [hep-th]].

[10] M.Yu. Kalmykov, V.V. Bytev, B.A. Kniehl, B.F.L. Ward, S.A. Yost, PoS ACAT08 (2008) 125 [arXiv:0901.4716 [hep-th]];

V.V. Bytev, M. Kalmykov, B.A. Kniehl, B.F.L. Ward, S.A. Yost, Proceedings of the International Linear Collider Workshop 2008 (LCWS08 and ILC08), University of Illinois at Chicago, 16-20 November 2008, edited by M. Barone, Y. Torun, N. Varelas, arXiv:0902.1352 [hep-th];

S.A. Yost, V.V. Bytev, M.Yu. Kalmykov, B.A. Kniehl, B.F.L. Ward, PoS ICHEP2010 (2010) 135 [arXiv:1101.2348 [math-ph]].

[11] V.V. Bytev, M.Yu. Kalmykov, B.A. Kniehl, arXiv:1105.3565 [math-ph].

[12] N. Takayama, Japan J. Appl. Math. 6 (1989) 147;

N. Takayama, J. Symbolic Comput. 20 (1995) 637.

[13] R. Mertig, R. Scharf, Comput. Phys. Commun. 111 (1998) 265 arXiv:hep-ph/9801383;

S.P. Martin, D.G. Robertson, Comput. Phys. Commun. 174 (2006) 133 arXiv:hep-ph/0501132.

[14] F. Jegerlehner, M.Yu. Kalmykov, Nucl. Phys. B 676 (2004) 365 arXiv:hep-ph/0308216.

[15] J. Fleischer, M.Yu. Kalmykov, Comput. Phys. Commun. 128 (2000) 531 arXiv:hep-ph/9907431.

[16] A.I. Davydychev, M.Yu. Kalmykov, Nucl. Phys. B $605 \quad$ (2001) 266 arXiv:hep-th/0012189.

[17] J. Fleischer, M.Yu. Kalmykov, Phys. Lett. $\quad$ B $470 \quad$ (1999) 168 arXiv:hep-ph/9910223.

[18] M. Czakon, M. Awramik, A. Freitas, Nucl. Phys. B (Proc. Suppl.) 157 (2006) 58 arXiv:hep-ph/0602029.

[19] F. Jegerlehner, M.Yu. Kalmykov, O. Veretin, Nucl. Phys. B 658 (2003) 49 arXiv:hep-ph/0212319;

A.I. Davydychev, M.Yu. Kalmykov, Nucl. Phys. B 699 (2004) 3 arXiv:hep-th/0303162. 